\title{
Retraction Note to: A Multi-agent Based Self-adaptive Genetic Algorithm for the Long-term Car Pooling Problem
}

Yuhan Guo $^{1,2}$ - Gilles Goncalves ${ }^{1,2}$ - Tienté Hsu ${ }^{1,2}$

The authors and the editor retract the above-mentioned article per the Committee on Publication Ethics (COPE) guidelines on plagiarism and self-plagiarism.

The article has used a substantial amount of the following paper without referencing:

- David Meignan, Abderrafiaa Koukam, Jean-Charles Créput Coalition-based metaheuristic: a self-adaptive metaheuristic using reinforcement learning and mimetism Journal of Heuristics (2010) 16:859 DOI: 10.1007/s10732-009-9121-7

Large sections of the article were also previously published by the same authors and not referenced:

- Yuhan Guo, Gilles Goncalves, Tienté Hsu A guided genetic algorithm for solving the long-term car pooling problem 2011 IEEE Workshop on Computational Intelligence In Production And Logistics Systems (CIPLS) DOI: 10.1109/CIPLS.2011.5953357

The authors apologize for any inconvenience they might have caused.

The online version of the original article can be found at http://dx.doi.org/10.1007/s10852-012-9175-7.

Yuhan Guo

jonguo.fr@gmail.com

1 Université Lille Nord de France, 59000, Lille, France

2 UArtois, LGI2A, 62400, Béthune, France 